\title{
Níveis de cálcio e granulometrias do calcário para frangas e seus efeitos sobre a produção e qualidade de ovos ${ }^{1}$
}

\section{Adriano Geraldo², Antônio Gilberto Bertechini ${ }^{3}$, Reinaldo Kanji Kato², Jerônimo Ávito Gonçalves de Brito ${ }^{2}$, Édison José Fassani ${ }^{4}$}

\footnotetext{
1 Parte da dissertação do primeiro autor. Projeto financiado pela FAPEMIG

2 Doutorando do Departamento de Zootecnia da UFLA.

${ }^{3}$ Departamento de Zootecnia da UFLA.

${ }^{4}$ Departamento de Zootecnia da UNIFENAS
}

\begin{abstract}
RESUMO - Realizou-se um experimento com o objetivo de avaliar os efeitos dos níveis nutricionais de cálcio e das granulometrias do calcário em rações para frangas nas fases de cria e recria sobre a produção de ovos. Utilizaram-se 600 poedeiras da linhagem Lohmann LSL, alimentadas nos períodos de cria e recria com rações experimentais suplementadas com fitase (500 FTU $/ \mathrm{kg})$ e calcário em granulometrias fina $(\mathrm{DGM}=0,135 \mathrm{~mm})$ e grossa $(\mathrm{DGM}=0,899 \mathrm{~mm})$ para atender os níveis de 0,60 0,$75 ; 0,90 ; 1,05$ e $1,20 \%$ de Ca total na ração. Os tratamentos foram dispostos em esquema fatorial 2 x 5 , com cinco repetições e 12 aves por unidade experimental e quatro períodos de 21 dias. Na fase de postura (18 a 30 semanas de idade), as aves receberam a mesma ração com 3,60\% de Ca. O nível de $0,60 \%$ de $\mathrm{Ca}$ e as granulometrias do calcário fornecido nas fases de cria e recria foram suficientes para proporcionar bom desempenho na postura e não afetaram a qualidade externa e interna dos ovos no período estudado.
\end{abstract}

Palavras-chave: poedeira, nutrição de aves, granulometria, calcário

\section{Effects of calcium levels and particle sizes of limestone on egg production and quality in pullets}

\begin{abstract}
This experiment was conducted to evaluate the effects of calcium levels and particle sizes of limestone on egg production. Six hundred Lohman LSL laying hens were randomly assigned to 10 treatments: two particle sizes of limestone $(\mathrm{DGM}=0.135 \mathrm{~mm}$ and $0.899 \mathrm{~mm})$ combined with five dietary calcium levels $(0.60,0.75,0.90,1.05$, and $1.20 \%$ $\mathrm{Ca})$, supplemented with phytase $(500 \mathrm{FTU} / \mathrm{kg})$. This experiment was analyzed as a 2 x 5 factorial arrangement with five replicates, with 12 hens per experiment unit and four periods of 21 days each. During the laying period (18 to 30 weeks of age), the hens were fed the same diet containing 3.6\% Ca. Both particle sizes tested and $0.60 \%$ calcium level showed good results of performance in the laying period with no effect on internal or external egg quality.
\end{abstract}

Key Words: hen, limestone, particle size, poultry nutrition

\section{Introdução}

O desenvolvimento genético nos últimos anos tem alterado aspectos fisiológicos importantes nas aves de postura, havendo a necessidade de novas pesquisas para equacioná-los, pois, tendo em vista a maior demanda na eficiência produtiva, existe hoje a necessidade de se produzir frangas de reposição que possam refletir, na sua fase de produção, todo o seu potencial genético para máxima eficiência produtiva.

Vários trabalhos têm sido realizados visando avaliar a granulometria ideal do calcário a ser fornecido para poedeiras para melhor desempenho e qualidade dos ovos, sendo consenso que maiores partículas da fonte de cálcio proporcionam maior retenção na moela, disponibilizando o cálcio vagarosa e uniformemente durante o período de formação da casca do ovo (Leeson \& Summers, 1997) e aumentando a retenção nos ossos medulares das poedeiras (Rao \& Roland, 1990). Para frangas de reposição, no entanto, não foram encontrados na literatura trabalhos avaliando efeitos das granulometrias da fonte de cálcio sobre o posterior desempenho produtivo.

O conhecimento dos níveis ideais de cálcio para cada fase do desenvolvimento da ave é de suma importância, visto que este macromineral é essencial para a formação e manutenção do esqueleto e que seu excesso na dieta interfere na disponibilidade de outros minerais, como fósforo, magnésio, manganês e zinco, além de tornar a ração menos palatável e diluir outros componentes quando altos níveis da fonte carbonato de cálcio (calcário) são utilizados. 
Estudos com cálcio $(\mathrm{Ca})$ e fósforo $(\mathrm{P})$ têm sido realizados com base no ganho de peso, na conversão alimentar, no teor de cinzas e na resistência óssea à quebra (Bailey et al., 1986), observando-se que o tamanho esquelético obtido no desenvolvimento da franga reflete as reservas de Ca disponíveis durante o pico de produção de ovos (Coelho, 2001).

Vários trabalhos foram realizados ao longo dos anos para estimativa da exigência nutricional de Ca para poedeiras. Persistem, no entanto, contradições quanto ao melhor nível a ser utilizado na dieta de frangas de reposição. Meyer et al. (1971) avaliaram o efeito do fornecimento de 0,$4 ; 0,7 ; 0,9 ; 1,2$ e 1,5\% de Ca na ração de aves da linhagem New Hampshire e Single Comb Leghorn branca, machos e fêmeas, no período de 8 a 21 semanas de idade e observaram que os níveis utilizados não tiveram efeitos sobre a mortalidade, o peso corporal e o comprimento do metatarso em ambos os sexos. O nível de $0,4 \%$ de Ca não foi adequado para o máximo crescimento ósseo e a resistência à quebra do metatarso, indicando ser o mínimo de $0,7 \%$ de Ca ideal para a máxima resistência óssea à quebra em ambos os sexos durante o período de crescimento.

O fornecimento de cinco diferentes níveis de $\mathrm{Ca}(0,60$ a $1,00 \% \mathrm{Ca}$ ) em rações para frangas de reposição leves e semipesadas no período de 7 a 12 semanas de idade não influenciou o consumo de ração e a resistência óssea (Vargas Jr. et al., 2001). O ganho de peso teve efeito quadrático para ambos os tipos de aves, de modo que o maior ganho de peso foi obtido nas frangas leves no nível de $0,799 \%$ de Ca e, nas semipesadas, no nível de $0,769 \%$ de Ca. Os níveis de 0,778 e $0,814 \%$ de Ca proporcionaram melhor conversão alimentar nas frangas leves $(\mathrm{CA}=3,9)$ e semipesadas $(C A=3,50)$, respectivamente, o que foi atribuído exclusivamente ao ganho de peso.

Segundo Coelho (2001), para a franga ter bom desenvolvimento esquelético, é necessário que a dieta contenha aproximadamente $1,00 \% \mathrm{Ca}$ e 0,45 a $0,50 \%$ de fósforo disponível.

Considerando a importância da formação óssea das frangas de reposição, o objetivo neste trabalho foi estudar o efeito de níveis nutricionais de Ca e de granulometrias do calcário na ração de frangas nas fases de cria e recria sobre o desenvolvimento corporal e a produção de ovos no período de 18 a 30 semanas de idade.

\section{Material e Métodos}

O experimento foi conduzido no Setor de Avicultura do Departamento de Zootecnia da Universidade Federal de Lavras utilizando-se 600 aves da linhagem comercial
Lohmann-LSL com 18 semanas de idade. As aves foram criadas durante o período de cria e recria com dietas experimentais formuladas à base de milho e farelo de soja, suplementadas com minerais, vitaminas e fitase $(500 \mathrm{FTU} / \mathrm{kg}$ ração), sendo isoprotéicas, isocalóricas e isofosfóricas, de acordo com as recomendações do Manual de Criação e Manejo da linhagem Lohmann LSL (1999).

Os tratamentos utilizados anteriormente, nas fases de cria e recria, constituíram um fatorial $2 \times 5$, com dez tratamentos, em um delineamento inteiramente casualisado, com cinco repetições, sendo estudada uma fonte de calcário em duas granulometrias $(\mathrm{DGM}=0,135 \mathrm{e} \mathrm{DGM}=0,899 \mathrm{~mm}) \mathrm{e}$ cinco níveis $(0,60 ; 0,75 ; 0,90 ; 1,05$ e 1,20\%) de cálcio. Após os períodos de cria e recria, ao atingirem 5\% de produção, as aves receberam uma mesma ração de postura durante 18 a 30 semanas de idade, à base de milho e farelo de soja, com $3,6 \% \mathrm{Ca}$, formulada de acordo com as recomendações de Leeson \& Summers (2001), sendo isoprotéica (17\% PB), isocalórica $(2.800 \mathrm{kcal} \mathrm{EM} / \mathrm{kg})$ e isofosfórica $(0,40 \%$ de $\mathrm{P}$ disponível).

As parcelas experimentais no período de postura consistiram de 12 aves e cinco repetições, sendo avaliadas as características produção média de ovos (\%/ave/dia), consumo de ração (g/ave/dia), peso médio dos ovos (g), conversão alimentar (por massa de ovos), perdas (\%), qualidade externa (peso específico, espessura da casca e porcentagem de casca) e interna (unidade Haugh), avaliadas em esquema de parcela subdividida no tempo, com quatro períodos de 21 dias.

Os resultados foram submetidos à análise estatística utilizando-se o Sistema de Análises de Variância para dados balanceados (SISVAR), descrito por Ferreira (2000), aplicando-se o teste $\mathrm{F}$ para granulometria e a análise de regressão para níveis de $\mathrm{Ca}$.

\section{Resultados e Discussão}

Não houve efeito da interação $(\mathrm{P}>0,05)$ granulometria do calcário $\times$ níveis de cálcio nas fases de cria e recria das frangas sobre a produção, o peso médio dos ovos, o consumo de ração, a conversão alimentar e a perda de ovos (Tabela 1).

Os resultados de produção média de ovos, de acordo com os níveis de Ca fornecidos nos períodos de cria e recria, estão de acordo com os obtidos por Berg et al. (1964) e Meyer et al. (1971), que também não observaram efeitos significativos dos níveis de Ca fornecidos no período de recria sobre a produção média de ovos. No entanto, a granulometria do calcário promoveu efeito significativo 
Tabela 1 - Produção média de ovos (\% por ave/dia), peso médio dos ovos (g), consumo de ração (g/ave/dia), conversão alimentar por massa de ovos $(\mathrm{g} / \mathrm{g})$ e porcentagem de perdas de ovos segundo os níveis de cálcio e as granulometrias do calcário fornecidos nas fases de cria e recria (média \pm erro-padrão)

Table 1 - Means of egg production (\%/hen/day), egg weight ( $g$ ), feed intake ( $g / h e n / d a y)$, feed:egg mass ratio (g/g) and percentage of eggs losses according to the dietary calcium levels and limestone particle sizes in the initial and growing phases (mean \pm standard error)

\begin{tabular}{|c|c|c|c|c|c|c|}
\hline \multirow[t]{2}{*}{$\begin{array}{l}\text { Granulometria do calcário }(\mathrm{mm}) \\
\text { Limestone particle size }(\mathrm{mm})\end{array}$} & \multicolumn{5}{|c|}{$\begin{array}{l}\text { Nível de cálcio (\%) } \\
\text { Calcium level (\%) }\end{array}$} & \multirow[b]{2}{*}{$\begin{array}{c}\text { Média }^{1} \\
\text { Mean }\end{array}$} \\
\hline & 0,60 & 0,75 & 0,90 & 1,05 & 1,20 & \\
\hline \multicolumn{7}{|c|}{$\begin{array}{l}\text { Produção de ovos }(\% \text { ave } / \text { dia }) \text { CV } 1=9,79 \% \\
\text { Egg production (\% hen/day) }\end{array}$} \\
\hline $\begin{array}{l}0,135 \\
0,899 \\
\text { Média (Mean) }\end{array}$ & $\begin{array}{c}87,48 \pm 1,92 \\
89,53 \\
88,53 \pm 1,36\end{array}$ & $\begin{array}{l}89,23 \\
83,53 \\
86,38\end{array}$ & $\begin{array}{l}89,29 \\
85,53 \\
87,41\end{array}$ & $\begin{array}{l}91,22 \\
86,58 \\
88,90\end{array}$ & $\begin{array}{l}88,17 \\
87,33 \\
87,75\end{array}$ & $\begin{array}{l}89,08 \pm 0,86 \mathrm{a} \\
86,50 \mathrm{~b}\end{array}$ \\
\hline \multicolumn{7}{|c|}{$\begin{array}{c}\text { Peso médio dos ovos }(\mathrm{g}) \mathrm{CV} 1=3,96 \% \\
\text { Average egg weight }(\mathrm{g})\end{array}$} \\
\hline $\begin{array}{l}0,135 \\
0,899 \\
\text { Média (Mean) }\end{array}$ & $\begin{array}{l}57,10 \pm 0,51 \\
58,20 \\
57,65 \pm 0,36\end{array}$ & $\begin{array}{l}57,35 \\
57,50 \\
57,42\end{array}$ & $\begin{array}{l}58,30 \\
57,85 \\
58,07\end{array}$ & $\begin{array}{l}58,60 \\
57,25 \\
57,92\end{array}$ & $\begin{array}{l}58,15 \\
56,85 \\
57,50\end{array}$ & $\begin{array}{l}57,90 \pm 0,23 \\
57,53\end{array}$ \\
\hline
\end{tabular}

\begin{tabular}{lcccccc}
\hline \multicolumn{7}{c}{$\begin{array}{c}\text { Consumo de ração (g/ave/dia) CV1 }=5,51 \% \\
\text { Feed intake (g/hen/day) }\end{array}$} \\
0,135 & $97 \pm 1,21$ & 98 & 100 & 100 & 98 & $96 \pm 0,54$ \\
0,899 & 99 & 98 & 98 & 97 & 96 & 97 \\
Média (Mean) & $98 \pm 0,85$ & 98 & 98 & 98 & 97 & \\
\hline
\end{tabular}

Conversão alimentar por massa de ovos $(\mathrm{g} / \mathrm{g}) \mathrm{CV} 1=9,12 \%$

Feed:egg mass ratio $(g / g)$

\begin{tabular}{|c|c|c|c|c|c|c|}
\hline 0,135 & $1,99 \pm 0,04$ & 1,98 & 1,98 & 1,89 & 1,95 & $1,96 \pm 0,02 \mathrm{a}$ \\
\hline 0,899 & 1,93 & 2,15 & 2,00 & 2,01 & 1,98 & $2,02 b$ \\
\hline Média $^{2}$ (Mean) & $1,96 \pm 0,03$ & 2,07 & 1,99 & 1,95 & 1,97 & \\
\hline \multicolumn{7}{|c|}{$\begin{array}{c}\text { Perda de ovos }(\%) \text { CV1 }=112,57 \% \\
\text { Eggs loss (\%) }\end{array}$} \\
\hline 0,135 & $0,90 \pm 0,18$ & 0,79 & 0,58 & 0,95 & 0,66 & $0,78 \pm 0,08$ \\
\hline 0,899 & 0,68 & 0,75 & 0,58 & 0,65 & 0,64 & 0,66 \\
\hline Média (Mean) & $0,79 \pm 0,13$ & 0,77 & 0,58 & 0,80 & 0,65 & \\
\hline
\end{tabular}

${ }^{1}$ Médias seguidas por letras diferentes na coluna diferem $(P<0,05)$ pelo teste $F$.

2 Efeito cúbico $(P<0,05)$.

${ }_{1}^{1}$ Means followed by different letters in the column differ $(P<0.05)$ by $F$ test.

2 Cubic effect $(P<0.05)$.

$(\mathrm{P}<0,05)$, de modo que o uso da granulometria fina $(\mathrm{DGM}=0,135 \mathrm{~mm})$ nas fases de cria e recria foi favorável, proporcionando maior produção média de ovos na fase de produção estudada. A não-sincronização da produção de ovos no início de postura pode ter causado esta diferença, que não era esperada, pois as aves nos períodos de cria e recria tiveram desempenho e características corporais e morfométricas do trato digestório semelhante para ambas as granulometrias.

Houve efeito dos períodos sobre a produção de ovos (Tabela 2), que foi menor no período 1, provavelmente porque as aves estavam no início do ciclo de postura. A partir do segundo período, verificou-se aumento da produção de ovos e, a partir do terceiro período, as aves atingiram o pico de postura, que se manteve até o final do experimento.
Verificou-se interação significativa $(\mathrm{P}<0,01)$ níveis de $\mathrm{Ca} \times$ períodos estudados sobre a produção de ovos (Tabela 3 ) e a conversão alimentar (Tabela 4). Os resultados indicaram diferenças nestas variáveis somente no primeiro período, quanto houve efeito cúbico dos níveis de Ca. Esse efeito pode estar relacionado à não-sincronização da produção no início da postura, estando presente também na variável conversão alimentar por estar relacionada à produção de ovos. Analisando períodos dentro de cada nível de $\mathrm{Ca}$, observa-se menor produção de ovos no período 1 , não ocorrendo diferenças entre os demais.

Os níveis de $\mathrm{Ca}$ e as granulometrias do calcário não afetaram o peso dos ovos, corroborando os dados reportados por Berg et al. (1964), que também não observaram efeitos dos níveis de 0,$66 ; 1,12$ e $2,01 \%$ de $\mathrm{Ca}$ fornecidos no período de 8 a 21 semanas de idade sobre o peso dos ovos. 
Tabela 2 - Produção média de ovos (\%/ave/dia), peso dos ovos $(\mathrm{g})$, consumo de ração $(\mathrm{g})$, conversão alimentar (CA) (g ração/g ovos) e perda de ovos (\%) segundo os períodos experimentais (média \pm erro-padrão)

Table 2 - Means of egg production (\%/hen/day), egg weight ( $g$ ), feed intake (g), feed:egg ratio(F/E) ( $g$ feed/g eggs) and egg losses (\%) according to the experimental periods (mean \pm standard error)

\begin{tabular}{|c|c|c|c|c|c|}
\hline $\begin{array}{l}\text { Período } \\
\text { Period }\end{array}$ & $\begin{array}{c}\text { Produção de ovos } \\
(\% / \text { ave } / \text { dia })^{1} \\
\text { Egg production }(\% / \text { hen/day })\end{array}$ & $\begin{array}{l}\text { Peso dos ovos } \\
(\mathrm{g})^{1} \\
\text { Egg weight }(g)\end{array}$ & $\begin{array}{l}\text { Consumo de ração } \\
(\mathrm{g})^{1} \\
\text { Feed intake }(\mathrm{g})\end{array}$ & $\begin{array}{c}\mathrm{CA} \\
(\mathrm{g} \text { ração/g ovos })^{1} \\
F / E(g \text { feed } / g \text { eggs })\end{array}$ & $\begin{array}{c}\text { Perda de ovos } \\
(\%) \\
\text { Egg losses (\%) }\end{array}$ \\
\hline 1 & $68,58 \mathrm{c}(0,596)^{2}$ & $52,88 \mathrm{~d} \quad(0,1358)$ & $84 \mathrm{~d}(0,2763)$ & $2,42 b \quad(0,0215)$ & $0,66 \quad(0,0978)$ \\
\hline 2 & $91,75 b \quad(0,596)$ & $56,42 \mathrm{c} \quad(0,1358)$ & $96 \mathrm{c}(0,2763)$ & $1,87 \mathrm{a} \quad(0,0215)$ & $0,67 \quad(0,0978)$ \\
\hline 3 & $95,30 \mathrm{a}(0,596)$ & $60,34 b \quad(0,1358)$ & $104 b(0,2763)$ & $1,81 \mathrm{a} \quad(0,0215)$ & $0,63(0,0978)$ \\
\hline 4 & $95,52 \mathrm{a} \quad(0,596)$ & $61,22 \mathrm{a} \quad(0,1358)$ & $108 \mathrm{a}(0,2763)$ & $1,85 \mathrm{a} \quad(0,0215)$ & $0,92 \quad(0,0978)$ \\
\hline DMS & 2,20 & 0,5006 & 1,0178 & 0,0790 & 0,360 \\
\hline
\end{tabular}

${ }_{1}^{1}$ Médias seguidas por letras diferentes na coluna diferem $(P<0,01)$ pelo Teste Tukey.

2 Erro-padrão da média.

${ }_{1}^{1}$ Means followed by different letters in the column differ $(P<0.01)$ by Tukey test.

2 Standard errormean.

Tabela 3 - Produção média de ovos (\%/ave/dia), de acordo com os períodos e níveis de $\mathrm{Ca}$

Table 3 - Means of egg production (\%/hen/day) according to the experimental periods and calcium levels

\begin{tabular}{lcccc}
\hline \multirow{2}{*}{$\begin{array}{l}\text { Nível de cálcio (\%) } \\
\text { Calcium level (\%) }\end{array}$} & \multicolumn{4}{c}{$\begin{array}{c}\text { Produção de ovos } \\
\text { Egg production (\%) por period }\end{array}$} \\
\cline { 2 - 5 } & $1^{1}$ & 2 & 3 & 4 \\
\hline $060^{2}$ & $71,96 \mathrm{~b}$ & $91,15 \mathrm{a}$ & $95,28 \mathrm{a}$ & 95,64 \\
0,75 & $62,50 \mathrm{~b}$ & $92,26 \mathrm{a}$ & $94,88 \mathrm{a}$ & $95,87 \mathrm{a}$ \\
0,90 & $66,96 \mathrm{~b}$ & $91,71 \mathrm{a}$ & $95,32 \mathrm{a}$ & $95,64 \mathrm{a}$ \\
1,05 & $70,12 \mathrm{~b}$ & $93,37 \mathrm{a}$ & $95,60 \mathrm{a}$ & $96,51 \mathrm{a}$ \\
1,20 & $71,37 \mathrm{~b}$ & $90,24 \mathrm{a}$ & $94,44 \mathrm{a}$ & $94,96 \mathrm{a}$ \\
\hline
\end{tabular}

1 Efeito cúbico $(P<0,01)$.

2 Médias seguidas por letras diferentes na mesma linha são diferentes $(P<0,01)$ pelo teste Tukey.

${ }^{1}$ Cubic effect $(P<0.01)$.

${ }^{2}$ Means followed by different letters in the row differ $(P<0.01)$ by Tukey test.

Tabela 4 - Conversão alimentar (gramas de ração por gramas de ovos) de acordo com o período e os níveis de cálcio ${ }^{1}$

Table 4 - Feed:egg ratio(gram of diet per gram of eggs) according to the experimental periods and calcium levels

Nível de cálcio $(\%) \quad$ Conversão alimentar $(\mathrm{g} / \mathrm{g})$

Calcium level (\%) Feed:egg ratio $(\mathrm{g} / \mathrm{g})$

\begin{tabular}{lcccc} 
& 11 & 2 & 3 & 4 \\
\hline $060^{2}$ & $2,28 \mathrm{~b}$ & $1,89 \mathrm{a}$ & $1,86 \mathrm{a}$ & $1,82 \mathrm{a}$ \\
0,75 & $2,73 \mathrm{~b}$ & $1,86 \mathrm{a}$ & $1,85 \mathrm{a}$ & $1,83 \mathrm{a}$ \\
0,90 & $2,46 \mathrm{~b}$ & $1,86 \mathrm{a}$ & $1,84 \mathrm{a}$ & $1,79 \mathrm{a}$ \\
1,05 & $2,32 \mathrm{~b}$ & $1,85 \mathrm{a}$ & $1,84 \mathrm{a}$ & $1,80 \mathrm{a}$ \\
1,20 & $2,28 \mathrm{~b}$ & $1,91 \mathrm{a}$ & $1,86 \mathrm{a}$ & $1,83 \mathrm{a}$ \\
\hline
\end{tabular}

1 Efeito cúbico $(P<0,01)$.

2 Médias seguidas por letras diferentes na linha são diferentes $(P<0,01)$ estatisticamente pelo teste de Tukey.

${ }^{1}$ Cubic effect $(P<0.01)$.

2 Means followed by different letters in the row differ $(P<0.01)$ by Tukey test.

Por outro lado, Anderson (1967) observou maior peso dos ovos em frangas alimentadas com ração contendo 1,30\% de Ca no período de 11 a 22 semanas de idade em relação aos níveis de 0,65 e $2,60 \%$ de $\mathrm{Ca}$.

O período experimental influenciou o peso dos ovos $(\mathrm{P}<0,01)$, estando os resultados de acordo com os obtidos por Card \& Nesheim (1966) e North \& Bell (1990), que atribuíram este aumento do peso ao incremento no tamanho da gema e do albúmen no ovo com o avançar da idade das aves (Tabela 2).

O consumo de ração na fase de postura não foi afetado (Tabela 1), indicando que os níveis de Ca e as granulometrias do calcário fornecidos nas fases de cria e recria não afetam esta variável, pois houve aumento no consumo somente com o avançar da idade das aves (Tabela 2).

A granulometria do calcário fornecido nas fases de cria e recria influenciou a conversão alimentar das aves na fase de postura, de modo que a granulometria fina (DGM = $0,135 \mathrm{~mm}$ ) proporcionou melhor conversão (Tabela 1), atribuída à maior produção de ovos das aves. O período também influenciou a conversão alimentar. No primeiro período, as aves apresentaram pior conversão alimentar, comprovada pelo alto consumo e pela baixa produção nesta fase (Tabela 2).

Quanto à porcentagem de perdas, não houve efeito dos tratamentos nas fases de cria e recria e dos períodos sobre esta variável (Tabelas 1 e 2), o que diverge dos resultados descritos por Meyer et al. (1971), que observaram maior incidência de ovos sem casca e quebrados em frangas alimentadas com rações contendo 0,4 e 1,5\% de Ca no período de 8 a 21 semanas de idade, enquanto, em frangas alimentadas com $0,90 \%$ de cálcio, foi menor a incidência de ovos quebrados.

Não foi observada interação significativa $(\mathrm{P}>0,05)$ granulometria do calcário $\times$ níveis de $\mathrm{Ca}$ fornecido nas fases de cria e recria, nem mesmo efeitos isolados dessas variáveis sobre a espessura da casca, a porcentagem de casca, o peso específico, a Unidade Haugh e a viabilidade de produção (Tabela 5).

Berg et al. (1964) também não observaram efeito significativo dos níveis de 0,$66 ; 1,12$ e 2,01\% de Ca fornecido na recria ( 8 a 21 semanas de idade) sobre o peso específico dos ovos. 
Tabela 5 - Espessura média de casca $(\mathrm{mm})$, porcentagem média de casca (\%), peso específico $\left(\mathrm{g} / \mathrm{cm}^{3}\right)$, unidade Haugh média dos ovos e viabilidade do experimento (\%), segundo os níveis de cálcio e as granulometrias do calcário fornecidos nas fases de cria e recria (média \pm erro-padrão)

Table 5 - Means of shell thickness ( $\mathrm{mm}$ ), shell percentage (\%), specific weight ( $\left.\mathrm{g} / \mathrm{cm}^{3}\right)$, egg Haugh unit and experimental viability (\%) according to the dietary calcium levels and limestone particle sizes in the initial and growing phases (mean \pm standard error)

Granulometria do calcário $(\mathrm{mm})$

Limestone particle size ( $\mathrm{mm}$ )
Nivel de cálcio $(\%)$

Calcium level (\%)

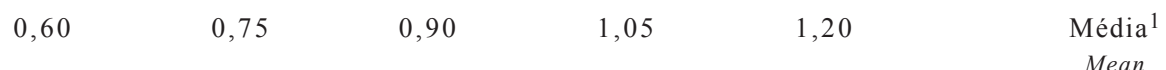

Espessura média de casca (mm) CV1=3,49\%

Mean shell thickness ( $\mathrm{mm}$ )

\begin{tabular}{lcccccc}
0,135 & $0,371 \pm 0,003$ & 0,371 & 0,372 & 0,366 & 0,372 & $0,370 \pm 0,001$ \\
0,899 & 0,368 & 0,369 & 0,375 & 0,368 & 0,371 & 0,370 \\
Média (Mean) & $0,370 \pm 0,002$ & 0,370 & 0,373 & 0,367 & 0,372 & \\
\hline
\end{tabular}

Porcentagem média de casca (\%) CV1 $=3,54 \%$

Mean shell percentage (\%)

$\begin{array}{lcccccc}0,135 & 9,52 \pm 0,08 & 9,51 & 9,50 & 9,34 & 9,47 & 9,47 \pm 0,03 \\ 0,899 & 9,38 & 9,49 & 9,62 & 9,48 & 9,61 & 9,52 \\ \text { Média (Mean) } & 9,45 \pm 0,05 & 9,50 & 9,56 & 9,41 & 9,54\end{array}$

Peso específico dos ovos $\left(\mathrm{g} / \mathrm{cm}^{3}\right) \mathrm{CV} 1=0,18 \%$

Mean egg gravity $\left(\mathrm{g} / \mathrm{dm}^{3}\right)$

\begin{tabular}{lcccccc}
0,135 & $1,087 \pm 0,0004$ & 1,086 & 1,086 & 1,085 & 1,086 & $1,086 \pm 0,0002$ \\
0,899 & 1,086 & 1,086 & 1,086 & 1,087 & 1,086 & 1,086 \\
Média (Mean) & $1,086 \pm 0,0003$ & 1,086 & 1,086 & 1,086 & 1,086 & \\
\hline
\end{tabular}

Unidade Haugh média dos ovos CV1 = 2,36\%

Mean egg Haugh unit

\begin{tabular}{lcccccc}
0,135 & $102,8 \pm 0,54$ & 103,4 & 103,2 & 103,4 & 102,7 & $103,1 \pm 0,24$ \\
0,899 & 102,9 & 102,2 & 102,8 & 103,9 & 102,9 & 102,9 \\
Média (Mean) & $102,8 \pm 0,39$ & 102,8 & 103,0 & 103,7 & 102,8 & \\
\hline
\end{tabular}

Viabilidade do experimento (\%) $\mathrm{CV}=3,03 \%$

Experiment viabilility (\%)

$\begin{array}{lcccccc}0,135 & 100,00 \pm 1,34 & 100,00 & 100,00 & 96,67 & 100,00 & 99,34 \\ 0,899 & 100,00 & 96,67 & 98,33 & 100,0 & 100,00 & 99,00 \\ \text { Média (Mean) } & 100,00 \pm 0,95 & 98,33 & 99,17 & 98,33 & 100,00\end{array}$

$\mathrm{Na}$ Tabela 6 são apresentados os valores médios de espessura média de casca, porcentagem de casca, peso específico $\left(\mathrm{g} / \mathrm{dm}^{3}\right)$ e Unidade Haugh dos ovos por período.

A menor espessura de casca obtida com o avançar da idade das aves está de acordo com resultados observados por outros autores, que também observaram diminuição da espessura da casca com o avançar da idade das aves, atribuída ao aumento do tamanho da gema e à deposição de albúmen (Card \& Nesheim, 1966; North \& Bell, 1990), não havendo incremento proporcional na deposição de casca. O mesmo pode ser observado para porcentagem de casca e peso específico, o que evidencia que a qualidade da casca do ovo é influenciada pela idade das aves, fato comumente verificado em outras pesquisas.

A qualidade interna piorou com o avançar da idade das aves, tendo em vista a pior qualidade de casca dos ovos, em função da redução da eficiência da poedeira, fato compro- vado pelos valores de Unidade Haugh ao longo do período experimental (Tabela 6 ).

Não foi observada interação $(\mathrm{P}>0,05)$ níveis de cálcio $\times$ granulometrias do calcário para o teor de cinzas e fósforo na tíbia na $30^{\mathrm{a}}$ semana de idade. Aves que receberam calcário na granulometria fina nas fases de cria e recria apresentaram maiores teores de fósforo na tíbia $(\mathrm{P}<0,05)$, não ocorrendo efeito dos níveis de cálcio. Os teores de cinzas não foram significativos $(\mathrm{P}>0,05)$ para granulometrias e níveis de cálcio, analisados independentemente (Tabela 7).

Verificou-se interação $(\mathrm{P}<0,01)$ níveis de cálcio $\times$ granulometrias do calcário para o teor de Ca na tíbia seca e desengordurada (Tabela 7), todavia, observou-se somente efeito de $4^{\circ}$ grau $(\mathrm{P}<0,01)$ para níveis de Ca dentro da granulometria grossa do calcário $(\mathrm{DGM}=0,899 \mathrm{~mm})$. Analisando as granulometrias dentro de níveis, houve efeito 
Tabela 6 - Espessura média de casca, porcentagem de casca, peso específico $\left(\mathrm{g} / \mathrm{cm}^{3}\right)$ e unidade Haugh média dos ovos por período (média \pm erro-padrão)

Table 6 - Means of shell thickness, shell percentage, specific gravity $\left(\mathrm{g} / \mathrm{cm}^{3}\right)$ and egg Haugh unit by period (means \pm standard error)

\begin{tabular}{lccc}
\hline $\begin{array}{l}\text { Período } \\
\text { Period }\end{array}$ & $\begin{array}{c}\text { Espessura de casca }(\mathrm{mm})^{1} \\
\text { Shell thickness }(\mathrm{mm})\end{array}$ & $\begin{array}{c}\text { Porcentagem de casca } \\
\text { Shell percentage }(\%)\end{array}$ & $\begin{array}{c}\text { Peso específico } \\
\left.\text { Specific gravity } \text { (g/cm }^{3}\right)^{l}\end{array}$ \\
\hline 1 & $0,371 \pm 0,001 \mathrm{~b}$ & $9,72 \pm 0,032 \mathrm{a}$ & $\begin{array}{c}\text { Unidade Haugh } \\
\text { Haugh unit }\end{array}$ \\
2 & $0,377 \pm 0,001 \mathrm{a}$ & $9,53 \pm 0,032 \mathrm{~b}$ & $1,089 \pm 0,0002 \mathrm{a}$ \\
3 & $0,367 \pm 0,001 \mathrm{bc}$ & $9,32 \pm 0,032 \mathrm{c}$ & $1,087 \pm 0,0002 \mathrm{~b}$ \\
4 & $0,366 \pm 0,001 \mathrm{c}$ & $9,39 \pm 0,032 \mathrm{c}$ & $1,085 \pm 0,0002 \mathrm{c}$ \\
DMS & 0,004 & 0,1191 & $1,084 \pm 0,0002 \mathrm{c}$ \\
\hline
\end{tabular}

${ }_{1}^{1}$ Médias seguidas por letras diferentes na coluna diferem $(P<0,01)$ pelo teste Tukey.

${ }^{1}$ Means followed by different letters in the column differ $(P<0.01)$ by Tukey test.

Tabela 7 - Teor de cinzas (\%), cálcio (\%) e fósforo na tíbia seca e desengordurada de poedeiras com 30 semanas de idade segundo os níveis de cálcio e as granulometrias do calcário fornecidos nas fases de cria e recria (média erro-padrão)

Table 7 - Contents of ash (\%), calcium (\%) and phosphorus (\%) in the dry tibia of hens with 30 weeks of age, according calcium levels and limestone particle size received by pullets in the initial and growing phases (means \pm standard error)

Granulometria do calcário $(\mathrm{mm}) \quad$ Nível de cálcio (\%)

Limestone particle size $(\mathrm{mm})$

Calcium level (\%)

\begin{tabular}{|c|c|c|c|}
\hline 0,60 & 0,75 & 0,90 & 1,05 \\
\hline
\end{tabular}

Teor de cinzas na tíbia

Contents of tibia ash (\%)

$\begin{array}{lcccccc}0,135 & 62,09 \pm 0,68 & 62,78 & 62,00 & 61,88 & 62,48 & 62,25 \pm 0,31 \\ 0,899 & 61,46 & 62,44 & 62,66 & 61,80 & 61,78 & 62,03 \\ \text { Média (Mean) } & 61,78 \pm 0,48 & 61,61 & 62,33 & 61,84 & 62,13\end{array}$

Média (Mean)

Teor de Ca na tíbia ${ }^{2}(\%) \mathrm{CV}=3,14 \%$

Contents of tibia $\mathrm{Ca}^{2}(\%)$

$\begin{array}{lcccccc}0,135 & 23,72 \pm 0,33 & 23,78 & 22,97 \mathrm{~b} & 23,56 & 23,58 & 23,52 \pm 0,15 \\ 0,899^{3} & 22,93 & 23,22 & 24,53 \mathrm{a} & 23,03 & 23,17 & 23,38 \\ \text { Média (Mean) } & 23,32 \pm 0,23 & 23,50 & 23,75 & 23,94 & 23,38 & \end{array}$

Teor de $\mathrm{P}$ na tíbia (\%) $\mathrm{CV}=3,14 \%$

Contents of tibia phosphorus (\%)

\begin{tabular}{lcccccc}
0,135 & $11,18 \pm 0,19$ & 11,17 & 10,64 & 11,16 & 11,19 & $11,07 \pm 0,09 \mathrm{a}$ \\
0,899 & 10,63 & 11,00 & 10,99 & 10,48 & 10,85 & $10,79 \mathrm{~b}$ \\
Média (Mean) & $10,90 \pm 0,14$ & 11,08 & 10,82 & 10,82 & 11,02 & \\
\hline
\end{tabular}

${ }_{1}^{1}$ Médias seguidas por letra diferentes na coluna diferem $(P<0,05)$ pelo teste $F$.

2 Médias seguidas por letras diferentes na coluna diferem $(P<0,01)$ pelo teste $F$.

${ }^{3}$ Efeito de $4^{\circ}$ grau $(P<0,01)$.

1 Means followed by different letters in the column differ $(P<0.05)$ by $F$ test.

2 Means followed by different letters in the column differ $(P<0.01)$ by F test.

$34^{\text {th }}$ order effect $(P<0.01)$.

significativo $(\mathrm{P}<0,01)$ somente no nível de $0,90 \%$ de $\mathrm{Ca}$, sendo a granulometria mais grossa favorável ao maior teor de Ca na tíbia. Não foi encontrada explicação para este resultado, visto que não ocorreram efeitos dos níveis de $\mathrm{Ca}$ e das granulometrias do calcário sobre a porcentagem de cinzas e P na tíbia.

Não foi constatada interação significativa $(\mathrm{P}>0,05)$ níveis de cálcio $\times$ granulometrias do calcário sobre $\mathrm{o}$ desenvolvimento do trato digestório, o comprimento do intestino delgado, o desenvolvimento da moela e o comprimento do metatarso na $30^{\mathrm{a}}$ semana de idade (Tabela 8). Os níveis de $\mathrm{Ca}$ e as granulometrias do calcário fornecidos nas fases de cria e recria não foram suficientes para afetar a morfometria do trato digestório das aves, ocorrrendo apenas efeito dos níveis de $\mathrm{Ca}(\mathrm{P}<0,01)$ sobre o comprimento do metatarso. 
Tabela 8 - Porcentagem de trato digestório, comprimento do intestino delgado ( $\mathrm{cm} / \mathrm{kg}$ de peso vivo), porcentagens de moela e comprimento do metatarso ( $\mathrm{cm} / \mathrm{kg}$ de peso vivo) em poedeiras com 30 semanas de idade segundo os níveis de cálcio e as granulometrias do calcário fornecidos nas fases de cria e recria (média \pm erro-padrão)

Table 8 - Digestory tract percentage, small intestine length ( $\mathrm{cm} / \mathrm{kg}$ body weight), percentages of gizzard and metatarsus length (cm/ $\mathrm{kg}$ body weight) according to the dietary calcium levels and limestone particle sizes in hens at 30 weeks old in the initial and growing phases (mean \pm standard error)

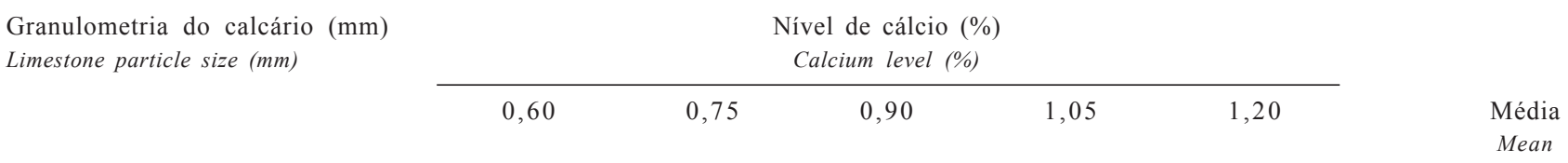

Porcentagem de trato digestório (\%) $\mathrm{CV}=9,33 \%$

Digestory tract percentage (\%)

\begin{tabular}{lcccccc}
0,135 & $7,7 \pm 0,33$ & 7,8 & 7,8 & 7,8 & 8,0 & $7,8 \pm 0,15$ \\
0,899 & 8,1 & 7,7 & 8,0 & 7,2 & 7,9 & 7,8 \\
Média (Mean) & $7,9 \pm 0,23$ & 7,7 & 7,9 & 7,5 & 8,0 & \\
\hline
\end{tabular}

Comprimento do intestino delgado $(\mathrm{cm} / \mathrm{kg}$ PV) $\mathrm{CV}=7,9 \%$

Small intestine length $(\mathrm{cm} / \mathrm{kg} \mathrm{BW})$

$\begin{array}{lcccccc}0,135 & 95,1 \pm 3,38 & 94,5 & 95,4 & 97,5 & 95,2 & 95,6 \pm 1,51 \\ 0,899 & 96,7 & 97,7 & 95,1 & 93,8 & 95,8 & 95,8 \\ \text { Média (Mean) } & 95,9 \pm 2,39 & 96,1 & 95,3 & 95,7 & 95,5\end{array}$

Porcentagem de moela (\%) CV2 $=12 \%$

Gizzard percentage (\%)

$\begin{array}{lcccccc}0,135 & 1,52 \pm 0,08 & 1,30 & 1,34 & 1,56 & 1,38 & 1,42 \pm 0,03 \\ 0,899 & 1,48 & 1,39 & 1,42 & 1,36 & 1,45 & 1,42 \\ \text { Média (Mean) } & 1,50 \pm 0,05 & 1,35 & 1,38 & 1,46 & 1,41 & \end{array}$

Comprimento do metatarso (cm/kg PV) $\mathrm{CV}=6,63 \%$

Metatarsus length $(\mathrm{cm} / \mathrm{kg} \mathrm{BW})$

$\begin{array}{lcccccc}0,135 & 5,9 \pm 0,18 & 6,3 & 5,9 & 6,3 & 6,3 & 6,1 \pm 0,08 \\ 0,899 & 5,9 & 6,4 & 5,7 & 6,2 & 6,5 & 6,1 \\ \text { Média }^{1} \text { (Mean) } & 5,9 \pm 0,13 & 6,4 & 5,8 & 6,3 & 6,4 & \end{array}$

1 Efeito de $4^{\circ}$ grau para níveis de Ca $(P<0,01)$.

$14^{\text {th }}$ order effect by calcium level $(P<0.01)$.

\section{Conclusões}

O nível de $0,60 \%$ de $\mathrm{Ca}$ e as granulometrias do calcário (DGM $=0,135$ e $0,899 \mathrm{~mm}$ ) em ração suplementada com 500 FTU's de fitase, fornecida nas fases de cria e recria, foram suficientes para o bom desempenho das aves na fase de postura (produção, conversão alimentar, peso médio dos ovos e porcentagem de perdas) e não afetaram a qualidade externa (peso específico, espessura e porcentagem de casca) e interna (Unidade Haugh) dos ovos nesta fase.

\section{Literatura Citada}

ANDERSON, D.L. Pre-laying nutritional and environmental factors in the performance of the adult fowl. Poultry Science, v.46, n.1, p.52-63, 1967.

ASSOCIATION OF OFFICIAL AGRICULTURAL CHEMISTSAOAC. Official methods of analysis. 15.ed. Arlington: Association of Official Analytical Chemists, 1990. n.1. 1117p.

BAILEY, C.A.; LINTON, S.; BRISTER, R. et al. Effects of gradel of dietary phosphorus on bone mineralization in the very young poult. Poultry Science, v.65, n.5, p.1018-1020, 1986.
BERG, L.R.; BEARSE, G.E.; MERRILL, L.H. The calcium and phosphorus requirements of White Leghorn pullets from 8-21 weeks. Poultry Science, v.43, n.4, p.885-896, 1964.

BORGES, A.L.C.C. Controle da ingestão de alimentos. Cadernos Técnicos da Escola de Veterinária da UFMG, v.27, p.6779, 1999

CARD, L.E.; NESHEIM, M.C. Poultry production. 10.ed. Philadelphia: Lea \& Febiger, 1966. 400p.

CHOI, J.H.; SO, B.S.; RYU, K.S. et al. Effects of pelleted or crumbled diets on the performance and the development of the digestive organs of broilers. Poultry Science, v.65, n.3, p.594-597, 1986.

COELHO, M. Early maturing layers require altered management. Feedstuffs, p.11-16, 2001.

FERREIRA, D.F. Sistema de análises de variância para dados balanceados. Lavras: UFLA, 2000. (SISVAR 4. 1. - pacote computacional).

VARGAS JR., J.G.; ALBINO, L.F.; ROSTAGNO, H.S. et al. Níveis nutricionais de cálcio para frangas de reposição leves e semipesadas de 7 a 12 semanas de idade. In: REUNIÃO DA SOCIEDADE BRASILEIRA DE ZOOTECNIA, 38., 2001, Piracicaba. Anais... Piracicaba: Sociedade Brasileira de Zootecnia, 2001. p.821-822.

KWAKKEL, R.P.; DUCRO, B.J.; KOOPS, W.J. Multiphasic analysis of growth of the body and its chemical components in White Leghorn pullets. Poultry Science, v.72, n.8, p.1421-1432, 1993.

LEESON, S.; SUMMERS, J.D. Commercial poultry nutrition. 2.ed. Guelph: University Books, 1997. 355p. 
LOHMANN. Manual de criação e manejo da linhagem Lohmann LSL. Uberlândia: Planalto, 1999.

MEYER, G.B.; BABCOCK, S.W.; SUNDE, M.L. Effect of various prelaying levels of dietary calcium upon subsequent performance in chickens. Poultry Science, v.50, n.2, p.536-547, 1971.

NORTH, M.O.; BELL, D.D. Commercial chicken production manual. 4.ed. New York: Champman \& Hall, 1990. 913p.

RAO, K.S.; ROLAND, D.A. In vivo limestone solubilization in commercial Leghorns: role of dietary calcium level, limestone particle size, in vitro limestone solubility rate, and the calcium status of the hen. Poultry Science, v.69, n.12, p.21702176, 1990 .

ROSTAGNO, H.S.; ALBINO, L.F.T.; DONZELE, J.L. et al. Tabelas brasileiras para aves e suínos: composição de alimentos e exigências nutricionais. Viçosa, MG: Universidade Federal de Viçosa, 2000. 141p.
SCHOULTEN, N.A. Níveis de cálcio em dietas para frangos de corte suplementadas com fitase. Lavras: Universidade Federal de Lavras, 2001. 79p. Dissertação (Mestrado em Zootecnia) - Universidade Federal de Lavras, 2001.

SIMCO, T.F.; STEPHENSON, E.L. Re-evaluation of the calciumphosphorus requirements of the chick. Poultry Science, v.40, n.5, p.1188-1192, 1961 